\title{
Outcomes of Prostate Cancer Screening by 5 alpha-Reductase Inhibitor Use
}

\section{Murtola, Teemu J.}

2017-08

Murtola , T J , Virkku, A, Talala , K, Stenman, U-H, Taari , K, Tammela , T L J \& Auvinen , A 2017 , ' Outcomes of Prostate Cancer Screening by 5 alpha-Reductase Inhibitor Use ' , Journal of Urology , vol. 198 , no. 2 , pp. 305-309 . https://doi.org/10.1016/j.juro.2017.02.069

http://hdl.handle.net/10138/297797

https://doi.org/10.1016/j.juro.2017.02.069

publishedVersion

Downloaded from Helda, University of Helsinki institutional repository.

This is an electronic reprint of the original article.

This reprint may differ from the original in pagination and typographic detail.

Please cite the original version. 


\title{
Outcomes of Prostate Cancer Screening by $5 \alpha$-Reductase Inhibitor Use
}

\author{
Teemu J. Murtola, ${ }^{*}, \dagger$ Anniina Virkku, Kirsi Talala, Ulf-Håkan Stenman, \\ Kimmo Taari, $\neq$ Teuvo L. J. Tammela§ and Anssi Auvinen\| \\ From the Faculty of Medicine and Life Sciences (TJM, AV, TLJT) and Faculty of Social Sciences (AA), University of Tampere and Department of Urology, \\ Tampere University Hospital (TJM, TLJT), Tampere and Finnish Cancer Registry (KT) and Departments of Clinical Chemistry (UHS) and \\ Urology (KT), University of Helsinki and Helsinki University Hospital, Helsinki, Finland
}

Purpose: Prostate cancer screening with prostate specific antigen reduces prostate cancer mortality but leads to over diagnosis of indolent prostate cancer. The use of $5 \alpha$-reductase inhibitors lowers prostate specific antigen and in theory could affect the performance of prostate specific antigen based screening. We evaluated the outcomes of prostate cancer screening in 5 $\alpha$-reductase inhibitors users.

Materials and Methods: The study was performed in FinRSPC (Finnish Randomized Study of Screening for Prostate Cancer). Of 80,454 men 31,866 were randomized to be screened at 4-year intervals during 1996 to 2004. Information on $5 \alpha$-reductase inhibitors reimbursements before prostate cancer during 1995 to 2009 was collected from the national prescription database for 78,615 men. We evaluated the effect of screening on prostate cancer risk and mortality by $5 \alpha$-reductase inhibitors using Cox regression.

Results: Men receiving $5 \alpha$-reductase inhibitors had higher median prostate specific antigen and were more often screen positive than nonusers. Despite this, screening did not significantly affect prostate cancer detection (HR $0.89,95 \%$ CI $0.79-1.01$ ) or mortality (HR $0.82,95 \%$ CI $0.51-1.32$ ) compared to findings in the control arm among men on $5 \alpha$-reductase inhibitors. On ROC analysis prostate specific antigen and age did not predict Gleason 7-10 prostate cancer as accurately in $5 \alpha$-reductase inhibitors users as it did among nonusers (first screening round AUC 0.79 vs 0.88 ).

Conclusions: Prostate specific antigen based screening among men receiving $5 \alpha$-reductase inhibitors did not improve the detection of high grade or metastatic prostate cancer, or prevent prostate cancer death.

Key Words: prostatic neoplasms, prostate-specific antigen, mass screening, $5 \alpha$-reductase inhibitors, Finland

Prostate cancer screening with serum PSA remains highly controversial despite extensive research and evidence of benefit in a large multicenter, randomized trial. Screening reduces PCa mortality ${ }^{1}$ but it also leads to substantial over diagnosis. ${ }^{1,2}$ Further, PSA is not a cancer specific biomarker. It is influenced by a number of other factors such as chronic prostatitis and $\mathrm{BPH}$, which cause false-positive screening results. Because it is unclear whether the benefits of prostate cancer

Abbreviations
and Acronyms
$5-\mathrm{ARI}=5 \boldsymbol{\alpha}$-reductase inhibitor
$\mathrm{BPH}=$ benign prostatic
hyperplasia
DRE $=$ digital rectal examination
FinRSPC $=$ Finnish Randomized
Study of Screening for Prostate
Cancer
PCa $=$ prostate cancer
PCPT = Prostate Cancer Preven-
tion Trial
PSA $=$ prostate specific antigen

Accepted for publication February 9, 2017

No direct or indirect commercial incentive associated with publishing this article.

The corresponding author certifies that, when applicable, a statement(s) has been included in the manuscript documenting institutional review board, ethics committee or ethical review board study approval; principles of Helsinki Declaration were followed in lieu of formal ethics committee approval; institutional animal care and use committee approval; all human subjects provided written informed consent with guarantees of confidentiality; IRB approved protocol number; animal approved project number.

* Correspondence: Department of Urology, Tampere University Hospital, Teiskontie 35, M-building, 3rd floor, PL 2000, 33521 Tampere, Finland (telephone: +358 3311 65015; e-mail: teemu.murtola@uta.fi).

$\dagger$ Financial interest and/or other relationship with Astellas, Janssen and MSD.

‡ Financial interest and/or other relationship with GlaxoSmithKline, AbbVie, Medivation, Astellas and Orion

$\S$ Financial interest and/or other relationship with Astellas, GlaxoSmithKline, Pfizer, Orion Pharma and Amgen.

|| Financial interest and/or other relationship with MSD and Epid Research. 
screening outweigh the harms, PCa screening is not recommended as a public health policy. However, it is possible that the efficacy of $\mathrm{PCa}$ screening could be improved by targeting screening to groups at increased risk for aggressive $\mathrm{PCa}$.

The 5-ARIs finasteride and dutasteride inhibit the conversion of testosterone into its active metabolite dihydrotestosterone, thereby decreasing prostate volume and serum PSA. ${ }^{3}$ Those 5-ARIs are used to treat $\mathrm{BPH}$ and finasteride is also prescribed for male pattern baldness. It was reported that 5-ARI decreases the $\mathrm{PCa}$ incidence compared to placebo while increasing the proportion of high grade tumors. $^{4,5}$ This was also confirmed in men who received these drugs to treat $\mathrm{BPH}^{6}{ }^{6}$ It is unclear whether the increased proportion of high grade tumors detected in men treated with a 5-ARI was due to more efficient diagnostics during 5-ARI therapy due to reduced prostate volume or to an absolute increase in the number of such tumors. Some studies suggest that 5-ARI improves the performance of PSA as a marker of high grade PCa by decreasing PSA elevations due to benign disease and even intraprostatic inflammation. ${ }^{6-8}$

The purpose of this study was to investigate the effect of $5 \alpha$-reductase inhibitor use on the outcomes of prostate cancer screening. We hypothesized that screening would be more specific for the detection of high grade tumors in 5-ARI treated men. We evaluated its possible modifying effect on $\mathrm{PCa}$ incidence and mortality in relation to screening.

\section{MATERIALS AND METHODS}

\section{Study Cohort}

FinRSPC is the largest component of ERSPC (European Randomized Study for Prostate Cancer Screening). It comprises 80,454 men born between 1929 and 1944 who were $55,59,63$ or 67 years old at study entry and living in the metropolitan areas of Helsinki and Tampere, Finland. The men were identified from the Finnish Population Register Centre. Those with a previous diagnosis of prostate cancer were identified from the Finnish Cancer Registry and excluded from the trial. During 1996 to 1999 men were randomly assigned to the screening arm $(31,866)$ or the control arm $(48,278)$. Men randomized to the control arm were not contacted but rather monitored through the Finnish Cancer Registry.

Men randomized to the screening arm were sent an invitation letter for a PSA screening test at 4-year intervals. A survey on prostate cancer family history and previous prostatic disease was included in the screening invitations. After written informed consent was provided a blood sample was drawn to determine serum PSA at a local Cancer Society of Finland clinic in Helsinki or Tampere. Invitations for PSA screening were repeated every 4 years but men older than 71 years were no longer invited.
Men with serum PSA $4 \mathrm{ng} / \mathrm{ml}$ or greater were referred to a local urological clinic for diagnostic evaluation comprising DRE, transrectal ultrasound and prostate biopsy. Men with PSA 3.0 to $3.99 \mathrm{ng} / \mathrm{ml}$ were referred for an additional test, including DRE during 1996 to 1998 and free-to-total PSA ratio determination with the cutoff at $16 \%$ since 1999. Men with a suspicious DRE or a freeto-total PSA ratio less than $16 \%$ were referred for further examination in the same manner as men with PSA $4 \mathrm{ng} / \mathrm{ml}$ or greater.

Prostate cancer was categorized by Gleason grade as low grade-Gleason 6 or less, or high/intermediate gradeGleason 7-10. Cases were additionally categorized as nonmetastatic or metastatic according to $\mathrm{M}$ stage at diagnosis.

Followup for cancer incidence and mortality started at randomization from 1996 to 1999 and ended at death, emigration from Finland or the common closing date of followup on December 31, 2014, whichever was first. Information on vital status and place of residence was obtained from the Population Register Centre. Information on cause of death was obtained from Statistics Finland. Deaths of men with PCa during 1996 to 2003 were reviewed by a cause of death committee to validate the official causes of death with excellent concordance. ${ }^{2}$

\section{Information on $\mathbf{5} \alpha$-Reductase Inhibitor Use}

Information on medication reimbursements during 1995 to 2009 was collected from the prescription database of SII (Social Insurance Institution of Finland). SII provides reimbursements for the cost of physician prescribed medicines as part of National Health Insurance to all Finnish citizens. All reimbursements for a purchase of reimbursable prescription drugs are recorded in the database. Data from the prescription database were obtained on 78,615 men, representing $97.7 \%$ of the FinRSPC study cohort.

All 5-ARIs in clinical use to treat BPH in Finland are available only by physician prescription and they are thereby reimbursable and registered in the database. During the study period the licensed $5 \alpha$-reductase inhibitors were finasteride (entire study period) and dutasteride (since 2005). The 5-ARIs prescribed to treat male pattern baldness were not reimbursable and, thus, not recorded in the prescription database.

\section{Statistical Analysis}

Cox proportional hazards regression was used to calculate $\mathrm{HRs}$ and 95\% CIs for prostate cancer risk and disease specific mortality in men in the screening arm compared to men in the control arm.

In the main analysis we evaluated 5-ARI effects before prostate cancer diagnosis on the efficacy of PSA screening by comparing the screening and control arms separately among 5-ARI users and nonusers. On analysis stratified by screening round we estimated the effect of being on 5-ARI prior to screening or the corresponding date in the control arm. Therefore, we compared the effects of screening in men on 5-ARIs and similarly among nonusers. Additionally, men treated with 5-ARI were stratified by the amount and duration of medication. 
PSA concentration as a predictive marker of $\mathrm{PCa}$ by stage and grade was evaluated by ROC analysis. The AUC was calculated separately among 5-ARI users and nonusers by comparing a model including age only to the model including PSA and age in each screening round.

Statistical analyses were performed using IBM ${ }^{\circledR}$ SPSS ${ }^{\circledR}$ Statistics 21.

\section{RESULTS}

\section{Population Characteristics}

The study population included 9,316 men who were 5-ARI users (11.9\%) and 69,299 nonusers (supplementary table 1, http://jurology.com/). Median age at baseline was 63 and 59 years, respectively. The proportion of screen-positive men was higher among 5-ARI users than nonusers (983 or $10.6 \%$ vs 3,239 or $4.7 \%$, p for difference $<0.001$ ). Of those receiving 5-ARI 1,131 (12.1\%) were diagnosed with $\mathrm{PCa}$, as were 7,122 not receiving 5-ARI (10.3\%). A total of $98(1.1 \%)$ and 736 men $(1.1 \%)$ died of PCa, respectively ( $\mathrm{p}$ for difference $=0.93$ ).

Compared to 5-ARI users the overall median PSA values were significantly lower among nonusers at each screening round (supplementary table 1, http:// jurology.com/). However, among men with PCa the median PSA at diagnosis was lower in those treated with 5-ARI for all tumor grades.

\section{Effect of Screening by $\mathbf{5} \alpha$-Reductase Inhibitor Use} Prostate Cancer Risk. Screening increased the HR of prostate cancer diagnosis compared to the control arm in 5-ARI nonusers but not users (HR 1.19, 95\% CI $1.14-1.25$ vs HR 0.89 , 95\% CI $0.79-1.01$, p for interaction <0.001). The risk decreased among men on 5-ARI in inverse correlation with the cumulative amount and duration of medication (supplementary table 2, http://jurology.com/).

The risk modifying effect of 5-ARI mainly concerned low risk cancer. Screening increased the detection of Gleason 6 or less and localized tumors in 5-ARI nonusers (both $\mathrm{p}$ for interaction $<0.001$ ), whereas among users no difference was observed in the FinRSPC study arm. However, the effect of screening on the risk of high grade and metastatic $\mathrm{PCa}$ did not differ by receipt of 5-ARI (supplementary table 2, http://jurology.com/).

Prostate Cancer Mortality. PSA screening significantly lowered the risk of $\mathrm{PCa}$ death in 5-ARI nonusers but not users (HR 0.60 , 95\% CI $0.51-0.71$ vs HR 0.82 , 95\% CI 0.51-1.32, table 1). However, the effect modification by 5-ARI use was statistically nonsignificant ( $\mathrm{p}$ for interaction 0.12).

When stratified by the cumulative amount and duration of 5-ARI therapy, the HR of prostate cancer death tended to increase in men on long-term
Table 1. Effect of PSA based screening on prostate cancer mortality by 5-ARI use in 78,615 men in FinRSPC

\begin{tabular}{ll}
\hline & $\begin{array}{c}\text { Screening vs Control } \\
\text { PCa Death HR (95\% CI) }\end{array}$ \\
\hline $\begin{array}{l}\text { 5-ARI use: } \\
\text { Yes }\end{array}$ & $0.82(0.51-1.32)$ \\
No & $0.60(0.51-0.71)$ \\
$\quad$ p for Interaction & 0.12 \\
Cumulative 5-ARI dose tertile: & \\
1 (28-240) & $0.51(0.22-1.17)$ \\
2 (241-1,090) & $0.79(0.36-1.75)$ \\
$3(1,091$ or greater) & $1.64(0.64-4.18)$ \\
5-ARI duration tertile: & \\
1 & $0.62(0.32-1.20)$ \\
2 & $1.02(0.40-2.60)$ \\
3 & $1.36(0.44-4.18)$ \\
\hline
\end{tabular}

* Daily dose is $5 \mathrm{mg}$ for finasteride and $0.5 \mathrm{mg}$ for dutasteride.

5-ARI (table 1). However, statistical power was limited in the stratified analyses.

\section{Prostate Specific Antigen as Prostate Cancer Grade and Stage Predictor in 5 $\alpha$-Reductase Inhibitor Users}

PSA and age were less accurate for predicting tumor Gleason grade among 5-ARI users than nonusers at all 3 screening rounds. At all screening rounds the AUCs of PSA and age as predictors of tumor stage were also lower in men receiving vs not receiving 5-ARI (table 2).

\section{Sensitivity Analysis}

When each screening round was analyzed separately, PSA screening increased the prostate cancer incidence in 5-ARI nonusers but not in users in the first 2 rounds (table 3 ). No difference was observed in the third screening round. A risk difference was observed only for Gleason 6 tumors at the second screening round.

\section{DISCUSSION}

Compared to 5-ARI nonusers PSA and the resulting proportion of screen-positive men were higher among 5-ARI users. Despite this, prostate cancer detection did not differ between the screening and

Table 2. PSA as predictor of prostate cancer grade and stage by 5 -ARI use in 3 consecutive screening rounds in 78,615 men in FinRSPC

\begin{tabular}{lccc}
\hline & \multicolumn{4}{c}{ Screening Round AUC (95\% CI)* } \\
\cline { 2 - 4 } 5-ARI Use before Round & 1 & 2 & 3 \\
\hline Gleason 7-10 PCa: & & & \\
$\quad$ None & $0.88(0.87-0.89)$ & $0.88(0.88-0.89)$ & $0.92(0.91-0.93)$ \\
$\quad$ Any & $0.79(0.70-0.88)$ & $0.78(0.72-0.85)$ & $0.84(0.79-0.91)$ \\
Metastatic PCa: & & & \\
$\quad$ None & $0.87(0.86-0.88)$ & $0.88(0.88-0.89)$ & $0.93(0.92-0.94)$ \\
Any & $0.73(0.64-0.82)$ & $0.78(0.73-0.84)$ & $0.82(0.77-0.88)$ \\
\hline
\end{tabular}

* Including age and PSA measured at round. 
Table 3. Effect of PSA based screening on prostate cancer incidence compared to nonscreening stratified by prescreening round 5-ARI use in 78,615 men in FinRSPC

\begin{tabular}{|c|c|c|c|}
\hline \multirow{2}{*}{$\begin{array}{l}\text { 5-ARI Use before } \\
\text { Screening Round }\end{array}$} & \multicolumn{3}{|c|}{ HR $(95 \%$ Cl) } \\
\hline & Overall PCa & Gleason 6 & Gleason 7-10 \\
\hline \multicolumn{4}{|l|}{ Round 1: } \\
\hline None & $1.60(1.45-1.77)$ & $1.97(1.74-2.22)$ & $1.30(1.07-1.59)$ \\
\hline Any & $0.78(0.43-1.42)$ & $0.93(0.46-1.88)$ & $0.45(0.13-1.62)$ \\
\hline$p$ for Interaction & 0.019 & 0.40 & 0.11 \\
\hline Round 2: & & & \\
\hline None & $1.31(1.20-1.42)$ & $1.72(1.55-1.92)$ & $0.84(0.72-0.97)$ \\
\hline Any & $0.72(0.51-1.00)$ & $0.81(0.52-1.24)$ & $0.61(0.36-1.04)$ \\
\hline $\begin{array}{l}p \text { for Interaction } \\
\text { Round 3: }\end{array}$ & 0.001 & 0.001 & 0.27 \\
\hline None & $1.13(1.04-1.23)$ & $1.49(1.31-1.69)$ & $0.92(0.82-1.03)$ \\
\hline Any & $1.02(0.80-1.31)$ & $1.22(0.83-1.77)$ & $0.88(0.63-1.23)$ \\
\hline p for Interaction & 0.43 & 0.31 & 0.79 \\
\hline
\end{tabular}

control arms in those on 5-ARI. Further, screening did not affect prostate cancer mortality in men receiving 5 -ARI, although the difference between users and nonusers was not significant. Elevated PSA levels are likely caused by underlying BPH, which indicated 5-ARI use. Men on 5-ARI have likely undergone some form of PSA testing as part of the diagnostic workup for $\mathrm{BPH}$, reducing the benefits achievable through systematic screening. Thus, our results suggest that 5-ARI users are probably not the ideal target group for PSA based prostate cancer screening.

On the other hand, receipt of 5-ARIs decreased the detection of nonmetastatic and Gleason $6 \mathrm{tu}-$ mors by screening but it did not affect the detection of Gleason 7-10 or metastatic tumors, or prostate cancer mortality. PCPT showed that 5-ARIs lower serum PSA in men with few or no lower urinary tract symptoms. ${ }^{4}$ Thus, presumably the decreased detection of localized and well differentiated tumors could have been due to a lower likelihood of being screen-positive, resulting in fewer prostate biopsies. However, the opposite was observed. Men on 5-ARIs to manage $\mathrm{BPH}$ had higher median PSA than nonusers and were more often screen-positive. This demonstrates that at the population level the PSA elevation caused by underlying $\mathrm{BPH}$ has more impact on the likelihood of being screen-positive than 5-ARI therapy.

There is another possible explanation. Because PSA testing is an integral part of $\mathrm{BPH}$ diagnostics, men on 5-ARI for BPH management had been tested for PSA at least once before the screening and, therefore, they formed a prescreened group. This may have diluted the effects of PSA based screening in this subgroup.

The risk of Gleason 6 or nonmetastatic prostate cancer in men in the FinRSPC screening arm decreased with longer duration of 5-ARI use. Thus, our results suggest that 5-ARI may decrease the over diagnosis of low risk tumors caused by benign causes of PSA elevation such as BPH and prostatic inflammation. If the finding was entirely caused by prescreening men on 5-ARI, there should have been no association with the duration of medication. The finding could reflect a reduced risk of low risk $\mathrm{PCa}$ with long-term 5-ARI use, as demonstrated in PCPT. ${ }^{4}$

Previous PCPT results suggested that 5-ARI could be administered to improve the performance of PSA as a marker of high grade prostate cancer. ${ }^{7,8}$ Our results do not support these findings since the risk of Gleason 7-10 disease was similar in the trial arms among 5-ARI users and nonusers. However, the difference could have been due to the dissimilarities of the study populations. In our study most men on 5-ARI have BPH whereas PCPT participants had little or no lower urinary tract symptoms at baseline. ${ }^{4}$ Unlike PCPT, our study did not include protocol mandated, end of study biopsies. Thus, the detection rate of indolent tumors was likely lower in our study.

Another difference compared to PCPT is that correction coefficients for PSA values among 5-ARI users were not routinely determined in our study. The criteria for screen positivity were similar regardless of 5-ARI treatment. Applying correlation coefficients when deciding to perform prostate biopsy was up to the discretion of the treating physician. This could potentially have caused differences in PCa detection between 5-ARI users and nonusers. However, it did not affect our comparisons of the screening and control arms in the 5-ARI user and nonuser groups.

Our results support previous studies showing no difference in prostate cancer mortality by 5 -ARI administration. ${ }^{9-12}$ Our findings suggest that screening may be more efficacious for decreasing prostate cancer mortality in men who are not receiving 5-ARIs, although the effect modification by 5 -ARI was not statistically significant. These drugs could be considered in future studies of targeted prostate cancer screening.

Overall the effect of screening on prostate cancer mortality was not as clear in FinRSPC as it was in the Rotterdam and Gothenburg sections of ERSPC. One likely explanation is the differing prevalence of opportunistic screening in the control group, although the differing prevalence of $\mathrm{BPH}$ and associated 5-ARI use may also have a role. As PSA measurement is part of the diagnostic workup of lower urinary tract symptoms, men with BPH represent a group with a high likelihood of undergoing screening outside the screening trial protocols.

A strength of our study is that it is founded on a large, population based, randomized trial of PSA 
based prostate cancer screening. We had the possibility of comparing outcomes at 3 screening rounds and, thus, evaluate the influence of repeat screening. The national prescription database provided extensive and accurate information on medication use. Since the information is collected routinely on all Finnish citizens, our results were not affected by recall bias.

The study also has some limitations. Because there was no randomization for 5-ARI use, the effect of $\mathrm{BPH}$ as the indication was difficult to discriminate from the effects of medication. Furthermore, information on the receipt of medication was based on medication purchases and actual medication use may have been lower.

\section{CONCLUSIONS}

In a randomized trial of PSA based prostate cancer screening the proportion of screen-positive men was higher among those treated with 5-ARIs. However, no difference in medication use was found in prostate cancer detection or mortality between the trial arms in this group.

\section{REFERENCES}

1. Schröder FH, Hugosson J, Roobol MJ et al: Prostate-cancer mortality at 11 years of follow-up. N Engl J Med 2012; 366: 981.

2. Kilpeläinen TP, Tammela TL, Malila $\mathrm{N}$ et al: Prostate cancer mortality in the Finnish randomized screening trial. J Natl Cancer Inst 2013; 105: 719 .

3. Marberger M: Drug Insight: 5alpha-reductase inhibitors for the treatment of benign prostatic hyperplasia. Nat Clin Pract Urol 2006; 3: 495.

4. Thompson IM, Goodman PJ, Tangen CM et al: The influence of finasteride on the development of prostate cancer. N Engl J Med 2003; 349: 215.

5. Andriole GL, Crawford ED, Grubb RL 3rd et al: Mortality results from a randomized prostate- cancer screening trial. N Engl J Med 2009; 360: 1310.

6. Murtola TJ, Tammela TL, Määttänen L et al: Prostate cancer incidence among finasteride and alpha-blocker users in the Finnish Prostate Cancer Screening Trial. Br J Cancer 2009; 101: 843.

7. Thompson IM, Chi C, Ankerst DP et al: Effect of finasteride on the sensitivity of PSA for detecting prostate cancer. J Natl Cancer Inst 2006; 98: 1128.

8. Murtola TJ, Gurel B, Umbehr M et al: Inflammation in benign prostate tissue and prostate cancer in the finasteride arm of the Prostate Cancer Prevention Trial. Cancer Epidemiol Biomarkers Prev 2016; 25: 463.
9. Azoulay L, Eberg $M$, Benayoun $S$ et al: $5 \alpha$-Reductase inhibitors and the risk of cancerrelated mortality in men with prostate cancer. JAMA Oncol 2015; 1: 314.

10. Preston MA, Wilson KM, Markt SC et al: $5 \alpha$ Reductase inhibitors and risk of high-grade or lethal prostate cancer. JAMA Intern Med 2014; 174: 1301

11. Kjellman A, Friis S, Granath $F$ et al: Treatment with finasteride and prostate cancer survival. Scand J Urol 2013; 47: 265.

12. Murtola TJ, Karppa EK, Taari $K$ et al: 5-Alpha reductase inhibitor use and prostate cancer survival in the Finnish Prostate Cancer Screening Trial. Int J Cancer 2016; 138: 2820. 